\title{
Correlation between sperm mitochondrial ND5 and ND6 gene variations and total fertilisation failure
}

\author{
Gen-Hong Mao, Xiao-Hui Huang, Xu-Jing Geng, Qian Li, Yu Zhang, Qian Dou
}

Reproductive Medical Center, The Second Affiliated Hospital of Zhengzhou University, Zhengzhou, Henan Province, China

Submitted: 20 May 2017

Accepted: 10 August 2017

Arch Med Sci 2020; 16 (3): 692-698

DOI: https://doi.org/10.5114/aoms.2020.94658

Copyright $\odot 2019$ Termedia \& Banach

\section{Abstract}

Introduction: The purpose of this study was to investigate the correlation between sperm mitochondrial NADH dehydrogenase subunit 5 (ND5) and NADH dehydrogenase subunit 6 (ND6) gene variations and total fertilisation failure (TFF).

Material and methods: A total of 232 sperm samples at the fresh in vitro fertilisation (IVF) cycle or the half-intracytoplasmic sperm injection (ICSI) cycle were collected for this retrospective controlled study on Han Chinese people between July 2011 and April 2014. Of the 232 total samples, 45 were from the IVF-TFF group and 187 were from couples with normal fertilisation (fertilisation rate $>50 \%$ ). The mitochondrial ND5 and ND6 gene variations and sperm haplotypes were confirmed using nested PCR and DNA sequencing.

Results: Ten homozygous variations were newly discovered, namely C12417T, T12441A, C12543A, C13650A, C13765A, T13769C, C13775T, A13776G, C13785A and C13845T. The gene variation rates of six sites, C12417T, C13650A, C13765A, T13769C, C13785A and C13845T, in the TFF group were significantly higher than those in the control group $(p<0.05)$. There were 231 heterozygous variations discovered; however, only nine heterozygous sites (12441, 12561, 12735, 13164, 13743, 13812, 13928, 14172 and 14368) had significantly higher gene variation rates than those in the control group $(p<0.05)$. In addition, the results showed that haplogroup $C$ did not affect TFF $(p>0.05)$, and the fertilisation failure rates of haplogroup $\mathrm{R}$ and haplogroup $\mathrm{D} 4 \mathrm{a}$ were both higher than those in the control group $(p<0.05)$.

Conclusions: Our results suggested that the ND5 and ND6 gene variations are correlated with TFF. Furthermore, this study indicated that haplogroup R and haplogroup D4a might be risk factors for TFF.

Key words: mitochondria, ND5, ND6, total fertilisation failure, variation.

\section{Introduction}

With the progress in assisted reproductive technology, the pregnancy rate following in vitro fertilisation (IVF) has significantly increased. However, fertilisation failure still exists, which is a frustrating experience for both patients and clinicians. The total fertilisation failure (TFF) refers to fertilisation failure in all oocytes. Patients with primary infertility and oligoasthenozoospermia might be at a higher risk of fertilisation failure [1]. A recent study showed that impaired sperm parameters were mainly involved in complete fertilisation failure in IVF and lower oocyte quality in intracytoplasmic sperm injection (ICSI) [2]. Fertilisation failure in IVF

\author{
Corresponding author: \\ Gen-hong Mao \\ Reproductive Medical \\ Center \\ The Second \\ Affiliated Hospital of \\ Zhengzhou University \\ Henan Province, \\ Zhengzhou 450014, China \\ Phone: 13733865362 \\ E-mail: \\ maogenhong2010@126.com
}


is mostly related to sperm abnormalities, whereas in ICSI, oocyte activation defects are the most frequent cause. TFF occurs at an estimated rate of $2-3 \%$ [3]. ICSI is a more effective treatment method for infertility resulting from male factors; however, there is still a failure rate of 1-3\% [4]. TFF is a very frustrating experience and causes enormous economic and emotional impacts on patients. In addition, clinicians also face significant mental stress. Studies have shown that if a couple experiences one TFF, the incidence of TFF in the following cycle is approximately 30\% [5]. Therefore, understanding the mechanism underlying TFF is important for preventing the occurrence of IVF failure. However, currently, little is known of the reasons for the development of TFF.

The ND complex provides $40 \%$ of the protons for ATP production in mitochondria [6]. It catalyses the transfer of the electrons in NADH to the oxygen molecules of electron receptors. Finally, energy produced during electron transfer is stored in the form of ATP [7]. It has been shown that point mutations, deletions and copy number changes in mitochondrial genes are associated with the activity of sperm. Our previous studies showed that variations in the mitochondrial gene ATP6 and mitochondrial haplotypes are correlated with IVF failure $[8,9]$. ND1-ND6 and ND4L in mitochondrial genes encode seven subgroups of mitochondrial respiratory chain complex I. It has been reported that the mitochondrial gene ND2 is highly conserved during the biological evolution process [10]. However, little is known about the association between ND gene variations and IVF failure.

Therefore, this study investigated the correlation between ND5 and ND6 gene variations and TFF to clinically predict IVF failure.

\section{Material and methods}

\section{Study subjects}

The patient numbers were inspired by a previous study [11], and a total of 232 sperm samples at the fresh IVF cycle or the half-ICSI cycle were collected for this retrospective controlled study of Han Chinese people between July 2011 and April 2014. The indications for IVF or half-ICSI assisted reproductive technologies were infertility caused by female factors or unknown reasons. In addition, spermiograms were collected, and the mean sperm concentration, and percentage of sperm motility grade $A$ and $B$ were evaluated. There were 45 TFF couples (the mean age of the women was $29.31 \pm 0.71$ years, and the mean age of men was $31.29 \pm 0.84$ years) and 187 couples (the mean age of the women was $30.98 \pm 0.35$ years, and the mean age of the men was $32.60 \pm 0.41$ years) with normal fertilisation rates (>50\%) in the control group. Successful fertilisation was defined as the presence of sperm and egg pronuclei in oocytes within 16-18 h of fertilisation; the absence of two or more pronuclei in oocytes was considered TFF [11]. This study was approved by the Research Ethics Committee of Zhengzhou University. Informed consent was obtained from each of the couples in the study group and the control group.

\section{Extraction of sperm mtDNA}

After liquefaction, the sperm samples were centrifuged in double-density (40\% and $80 \%$ Pureception) gradient centrifugation fluid. The sperm with different activities were separated in the gradient centrifugation fluid based on their different migration rates. Next, approximately $7 \times 10^{6}$ sperm were washed using 5\% 1026 and centrifuged at $200 \times \mathrm{g}$ for $8 \mathrm{~min}$. The supernatant was discarded and the precipitate was stored at $-80^{\circ} \mathrm{C}$ for DNA extraction. During DNA extraction, the precipitate was digested with $600 \mu$ l of lysis buffer containing $1 \%$ SDS and $0.2 \mathrm{mg} / \mathrm{ml}$ Proteinase $\mathrm{K}$. The mixture was incubated at $60^{\circ} \mathrm{C}$ for $40 \mathrm{~min}$ and then diluted with $800 \mu \mathrm{l}$ of pure ethanol and centrifuged at $11,100 \times \mathrm{g}$ for $1 \mathrm{~min}$. Finally, the isolated DNA precipitate was air dried before adding $50 \mu \mathrm{l}$ of $\mathrm{ddH}_{2} \mathrm{O}$.

\section{Nested PCR and sequence analysis}

The primer sequences, reaction conditions of the nested PCR and their GenBank accession numbers are shown in Table I. The first round of amplification was performed in a reaction mixture containing the following reagents: $2 \mathrm{mM} \mathrm{MgCl}$, $200 \mathrm{nM}$ of each dNTP, $20 \mathrm{nM}$ of each primer, $2 \mathrm{U}$ Taq DNA polymerase, $2 \mu \mathrm{l}$ DNA template and $\mathrm{ddH}_{2} \mathrm{O}$ to a final reaction volume of $50 \mu \mathrm{l}$. The second round of amplification was performed under similar conditions as the first round except the annealing temperature was different. The direct sequencing of both strains of genes in the samples was analysed using the Applied Biosystems (ABI, Foster City, CA) 3730 DNA Sequencing Analysis Gene Mapper Version 4.0. In the forward and reverse sequencing or in the twice repeated forward sequencing, if the second wave peak was higher than $10 \%$ of the wave peak at the same site, then the sequence was considered a heterozygous variant [12].

\section{Classification of mitochondrial gene haplogroup polymorphisms}

According to the human mitochondrial single nucleotide polymorphism (SNP) database (http:// www.mitomap.org/foswiki/bin/view/MITOMAP/ HaplogroupMarkers), three haplogroups were screened from the ND5 and ND6 genes. Haplogroup $\mathrm{N}$, which contained the C12705C SNP locus, was 
Table I. Nested PCR primers, reaction conditions and GenBank accession numbers

\begin{tabular}{|c|c|c|c|c|c|}
\hline Gene & Primers & $\begin{array}{l}\text { Product } \\
\text { [bp] }\end{array}$ & $\begin{array}{c}\text { Annealing } \\
\text { temperature }\left[{ }^{\circ} \mathrm{C}\right]\end{array}$ & No. of cycles & $\begin{array}{c}\text { Accession } \\
\text { numbers }\end{array}$ \\
\hline \multicolumn{6}{|l|}{ MT-ND5: } \\
\hline \multirow[t]{2}{*}{ Outer } & F:ACAACAGAGGCTTACGACC & 2316 & 53 & 30 & NC-012920 \\
\hline & R:GGGGAATGATGGTTGTCTT & & & & \\
\hline \multirow[t]{2}{*}{ Inner } & F:CGACCCCTTATTTACCGAGA & 2040 & 54 & 30 & NC-012920 \\
\hline & R:TGGGCGTTGATTAGTAGTAG & & & & \\
\hline \multicolumn{6}{|l|}{ MT-ND6: } \\
\hline \multirow[t]{2}{*}{ Outer } & F:CTTACGAGCCAAAACCTGC & 1027 & 55 & 30 & NC-012920 \\
\hline & R:GTAGCGGATGATTCAGCCA & & & & \\
\hline \multirow[t]{2}{*}{ Inner } & F:TACGAGCCAAAACCTGCCCC & 896 & 60 & 30 & NC-012920 \\
\hline & R:GCCAAGGAGTGAGCCGAAGT & & & & \\
\hline
\end{tabular}

the R subtype. In haplogroup M, the specific SNP locus of the C subtype was A13263G. The specific SNP locus in haplogroup D4a was A13651G.

\section{Sequence comparison}

The sequence results were compared with the human revised Cambridge reference sequence (rCRS) provided by the Mitomap database (www. mitomap.org). The detected nucleotide variation sites were confirmed using the peak map and it was determined whether the amino acids encoded by the variation sites had changed.

\section{Statistical analysis}

The continuous data were expressed as the mean \pm standard deviation. The categorical data were expressed as numbers and percentages. The measurement data were analysed using the independent sample $t$-test and the count data were analysed using the $\chi^{2}$ test or Fisher's exact test. All analyses were bilateral tests. $P$-values lower than 0.05 indicated that the differences were statistically significant.

\section{Results}

\section{General characteristics of total fertilisation failure and control groups}

There were no significant differences between the two groups in mean sperm concentration, percentage of sperm motility grade $\mathrm{A}$ or mean $\mathrm{FSH}$ and $\mathrm{LH}$ levels in women. However, the percentage of sperm with motility grade $B$, the number of retrieved oocytes and the number of metaphase II oocytes were markedly lower in the total fertilisation failure group than in the control group $(p<0.01)$. In addition, the fertilisation rates were
0 and $80.00 \%$, respectively, in the total fertilisation failure and control groups, and there was a significant difference between the two groups $(p<0.01)$ (Table II).

\section{Mitochondrial DNA variants and total fertilisation failure}

In this study, 168 homozygous variations were discovered in the ND5 gene, of which variations at 62 sites were statistically significant between the two groups $(p<0.05)$ and there were 47 missense variations. There were 22 homozygous variations discovered in the ND6 gene, of which variations at seven sites were statistically significant between the two groups $(p<0.05)$, and there were seven missense variations. Among the 69 homozygous variation sites with statistical significance in this study, only the C12705T site variation decreased the occurrence of TFF, whereas variations at other sites significantly increased the occurrence rate of TFF ( $p<0.05)$. In addition, five variation sites, G12528A, T12696C, G13466A, C13905T and $\mathrm{T} 14088 \mathrm{C}$, were present in at least three copies in the sperm samples from the TFF group, whereas variations at these sites were not found in the normal fertilisation group (Supplementary Table SI).

Ten homozygous variation sites in the ND5 gene were newly discovered in this study, namely C12417T, T12441A, C12543A, C13650A, C13765A, T13769C, C13775T, A13776G, C13785A and C13845T (Figure 1). The variation rates of six of the newly discovered sites, C12417T, C13650A, C13765A, T13769C, C13785A and C13845T, were significantly higher in the TFF group than in the control group $(p<0.05)$, which suggests that variations at these six sites might be associated with IVF failure. There were four missense variations among the ten newly discovered homozygous 
Table II. General characteristics of the total fertilisation failure and control subjects

\begin{tabular}{|c|c|c|c|}
\hline Variable & IVF failure group $(n=45)$ & Control group $(n=187)$ & $P$-value \\
\hline \multicolumn{4}{|l|}{ Sperm: } \\
\hline Concentration $\left[\times 10^{6} / \mathrm{ml}\right]$ & $55.46 \pm 4.06$ & $57.22 \pm 1.83$ & NS \\
\hline Motility grade A (\%) & $30.61 \pm 2.26$ & $30.11 \pm 0.79$ & NS \\
\hline Motility grade B (\%) & $19.82 \pm 1.40$ & $26.54 \pm 0.85$ & $<0.01$ \\
\hline \multicolumn{4}{|l|}{ Serum: } \\
\hline Female basal FSH [mIU/ml] & $6.35 \pm 0.52$ & $6.07 \pm 0.17$ & NS \\
\hline Female basal LH $[\mathrm{mlU} / \mathrm{ml}]$ & $5.54 \pm 0.73$ & $5.43 \pm 0.25$ & NS \\
\hline \multicolumn{4}{|l|}{ Oocyte, $n$ : } \\
\hline Retrieved oocytes & $8.49 \pm 0.82$ & $12.55 \pm 0.47$ & $<0.01$ \\
\hline Metaphase II oocytes & $7.40 \pm 0.66$ & $11.48 \pm 0.45$ & $<0.01$ \\
\hline Fertilisation rate & 0 & $0.80 \pm 0.01$ & $<0.01$ \\
\hline
\end{tabular}

$N S-$ not significant. Values are mean $\pm S D$.

variations. Specifically, the hydrophilic amino acid proline at the $\mathrm{C} 13765 \mathrm{~A}$ site changed to the hydrophilic amino acid threonine, and the hydrophilic amino acid phenylalanine at the T13769C site changed to the hydrophilic amino acid serine. The variations in the other six were all synonymous variations.

Significant missense variations were discovered at 18 sites in the ND5 gene including A12367G, A12469G, A12662G, G12940A, A12950G, G13145A, A13276G, G13466A, A13651G, C13712T, T13753C, C13754T, C13765A, T13769C, T13820C, C13934T, A14002G and A14053G. Only one significant missense variation was discovered in the ND6 gene, which was at the G14384 site (Supplementary Table SI). Protein function prediction was performed using the Uniport database (http:// www.uniprot.org/) and Polyphen-2 (http://genetics.bwh.harvard.edu/pph2). In the analysis results, if the score was closer to 1.0, the possibility of damage was higher, whereas if the score was closer to 0 , the possibility of damage was lower. The analysis results showed that A13651G missense variation was predicted to be probably damaging with a score of 0.996 , which suggested that the possibility of the A13651G missense variation to cause IVF failure was very high.

A total of 186 heterozygous variations were discovered in the ND5 gene, of which heterozygous variations at seven sites ( $T / G$ variation at $12441 \mathrm{~T}, \mathrm{G} / \mathrm{C}$ variation at $12561 \mathrm{G}, \mathrm{T} / \mathrm{G}$ variation at $12735 \mathrm{C}, \mathrm{A} / \mathrm{G}$ variation at $13164 \mathrm{~A}, \mathrm{~T} / \mathrm{C}$ variation at $13743 \mathrm{~T}, \mathrm{~T} / \mathrm{C}$ variation at $13812 \mathrm{~T}$ and $\mathrm{G} / \mathrm{A}$ variation at $13928 \mathrm{G}$ ) significantly decreased the IVF ability of sperm and caused the occurrence of IVF failure $(p<0.05)$. A total of 45 heterozygous variations were discovered in the ND6 gene, of which the $\mathrm{T} / \mathrm{C}$ variation at the $14172 \mathrm{~T}$ site and $\mathrm{C} / \mathrm{T}$ variation at the $14368 \mathrm{C}$ site also decreased the IVF ability of sperm $(p<0.05)$ (Supplementary Table SII).

Association between total fertilisation failure and mitochondrial DNA haplogroups C, R and D4a.

This study showed that haplogroup $C$ did not affect the IVF ability of sperm. The homozygous variation at the C12705T site was a beneficial variation for the IVF ability of sperm; however, the sperm of haplogroup $\mathrm{R}$ with the specific SNP locus at $\mathrm{C} 12705 \mathrm{C}$ were prone to TFF $(p<0.05)$ (Table III). The A13651 site variation in the ND5 gene was an important polymorphism site determining the haplogroup D4a in the Asian population. The protein function prediction results suggested that the risk of TFF significantly increased in the haplogroup D4a group.

\section{Discussion}

Previous studies have shown that the function of the mitochondria in humans is closely associated with infertility [8, 9, 13-16]. Zhu et al. [14] showed that through the PI3K and GSK $3 \alpha$ signalling pathways, the activation of Toll-like receptors damages the mitochondrial membrane of sperm and reduces mitochondrial ATP synthesis, thus resulting in a reduction in the fertilisation ability of sperm. Mi et al. [15] showed that Spata 19 influenced the fertilisation ability of sperm by influencing the mitochondrial structure and function of sperm. Condorelli et al. [16] showed that myoinositol increased the fertilisation ability of sperm in oligoasthenozoospermia patients by improving the mitochondrial function of sperm. Our previous studies showed that variations in the mitochondrial gene ATP6 and mitochondrial haplotypes are correlated with IVF failure [8, 9]. 
A

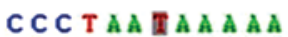

C12417T

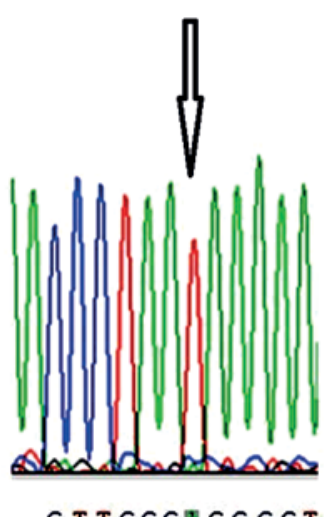

D

C T T C C C I G C C C T

C13650A

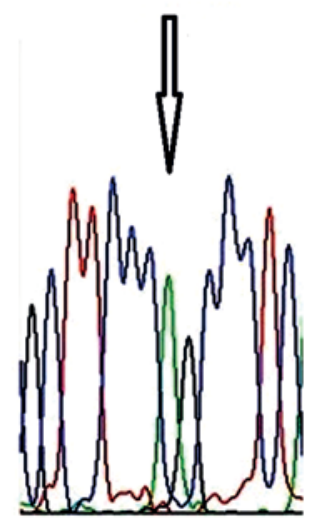

G

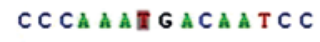

C13775T A13776G

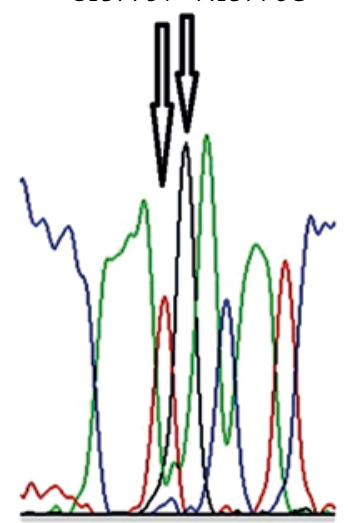

B

CA T TA I G T A A A

T12441A

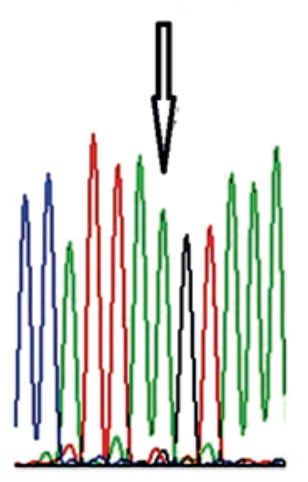

E a Ca T CC I C C C C C

C13765A

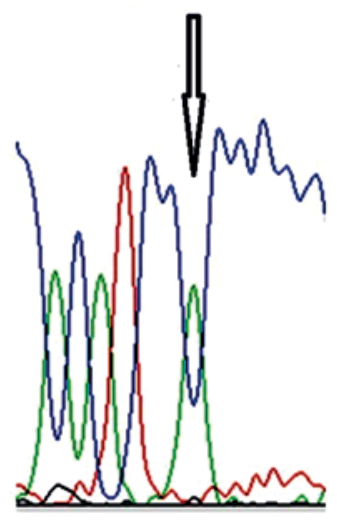

H A T C C CIIATT TA

C13785A

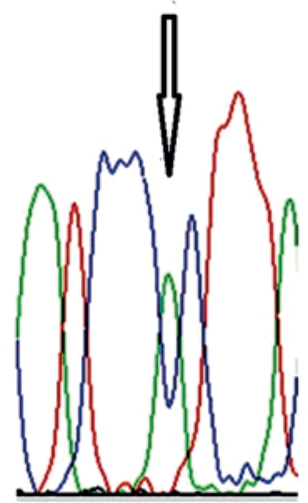

C
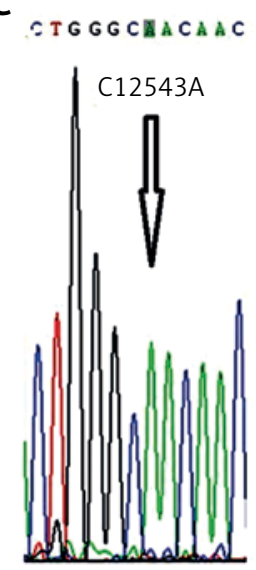

F c c c c canatg

T13769C

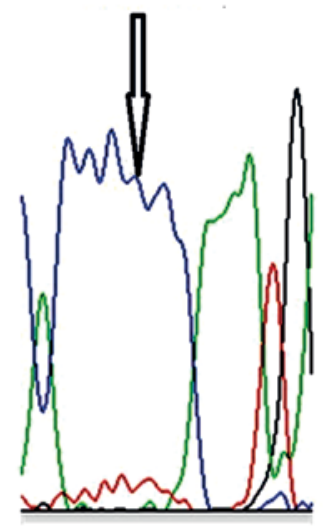

I C TAG AIICT CAA

C13845T

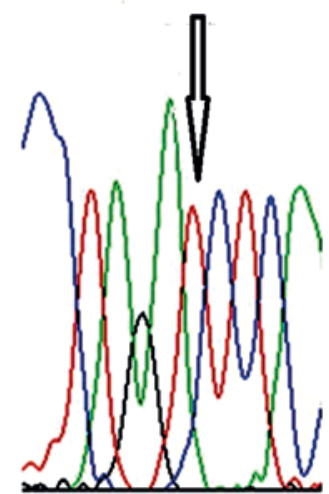

Figure 1. Identification of ten novel variations in ND5 gene

Previous literature reported that variation at the C12705T site of the ND5 gene might increase the risk of developing type 2 diabetes mellitus. However, among the 69 homozygous variation sites with statistical significance in this study, the C127057 site variation was found to be a protective variant against the occurrence of TFF. Variations at the other 68 sites were shown to significantly increase the occurrence rate of TFF $(p<0.05)$
(Supplementary Table SI). In addition, variations at five sites, G12528A, T12696C, G13466A, C13905T and T14088C, were present in at least three copies in the sperm samples from the TFF group, whereas variations at these sites were not discovered in the normal fertilisation group. Among them, only the G13466A site variation was a missense variation. In this variation, the second base of the AGC codon is changed to AAC, which changes the 
Table III. Comparisons of haplogroups in total fertilisation failure and controls

\begin{tabular}{|lcccc|}
\hline mtDNA haplogroup & Involved SNP & TFF (\%) $(n=45)$ & Controls (\%) $(n=187)$ & $P$-value \\
\hline \begin{tabular}{l} 
M subgroup: \\
\hline C
\end{tabular} & A13263G & $0(0.00)$ & $5(2.67)$ & NS \\
\hline N subgroup: & & & & \\
\hline R & C12705C & $25(55.56)$ & $73(39.04)$ & $<0.05$ \\
\hline D4a & A13651G & $2(4.44)$ & $0(0)$ & $<0.05$ \\
\hline
\end{tabular}

NS - not significant.

hydrophilic amino acid serine into the hydrophilic amino acid asparagine. The results suggested that variations at five sites, G12528A, T12696C, G13466A, C13905T and T14088C, might be associated with IVF failure.

Ten homozygous variation sites in the ND5 gene were newly discovered (Figure 1). The gene variation rates of six sites (C12417T, C13650A, C13765A, T13769C, C13785A and C13845T) in the TFF group were all significantly higher than those in the control group $(p<0.05)$. These results suggested that variations at these six sites might be associated with the occurrence of total IVF failure. Among the ten newly discovered homozygous variations, there were four missense variations, one of which involved a change of the hydrophobic amino acid proline at the C13765A site into the hydrophilic amino acid threonine and the other involved a change of the hydrophobic amino acid phenylalanine at the $\mathrm{T} 13769 \mathrm{C}$ site into the hydrophilic amino acid serine. Variations at the other six sites were all synonymous variations, which do not cause amino acid changes but can influence mRNA stability and protein structure, expression and function [17]. A previous study has shown that synonymous variations might also be involved in the development of various diseases [18].

Significant missense variations at 18 sites were discovered in the ND5 gene. There was only one missense variation discovered in the ND6 gene, which was at the G14384A site. Protein function prediction of these 17 missense variations using the Uniport database (http://www.uniprot. org/) and Polyphen-2 (http://genetics.bwh.harvard.edu/pph2) showed that the possibility of the A13651G variation site to cause IVF failure was very high. The A13651G site variation in the ND5 gene was a major polymorphism site determining the haplogroup D4a in the Asian population. The missense variation at this site changed the moderately conserved threonine into alanine, which altered the spatial structure of the transmembrane protein and influenced electron transfer in respiratory chain complex I, thus affecting mitochondrial OXPHOS and resulting in the occurrence of IVF failure.
This study showed that the occurrence rates of the heterozygous gene variations at the nine sites, 12441, 12561, 12735, 13164, 13743, 13812, 13928, 14172 and 14368, in the ND5 and ND6 genes were significantly higher than those in the normal fertilisation group, suggesting that heterozygous variations at these sites were correlated with TFF. Studies have shown that abnormalities in the ND complex might be associated with the development of mitochondrial encephalomyopathy, Parkinson's disease and Huntington's disease [19]. Mitochondria play very important roles in both spermatogenesis and sperm movement. The maintenance of the forward movement ability and fertilisation ability of sperm depends on the normal ratio of $N A D(P) H / N A D(P)$, the intracellular reactive oxygen species (ROS) balance and the transmembrane potential generated by the mitochondrial electron transfer chain [20, 21]. ND5 and ND6 gene variations might result in changes in the structure and function of the ND complex, the accumulation of NADH and the blockade of the OXPHOS process. As a result, the mitochondrial electron transfer chain may become overloaded due to the increase in the electron donor NADH, which results in an increase in the ratio of NAD $(P)$ $H / N A D(P)^{+}$, an increase in ROS and changes in the mitochondrial transmembrane potential in sperm. These changes influence the fertilisation ability of sperm and increase the occurrence of TFF.

This study showed that haplogroup $C$ did not influence the IVF ability of sperm. The homozygous variation at the C12705T site was a beneficial variation for IVF ability in sperm; however, the sperm of haplogroup R, with the specific SNP site at $\mathrm{C} 12705 \mathrm{~T}$, were more prone to TFF $(p<0.05)$. These results suggested that haplogroup $R$ might be a risk factor for IVF failure. The A13651G site variation in the ND5 gene was a major polymorphism locus determining haplogroup D4a in the Asian population. The protein function prediction results suggested that haplogroup D4a might also be a risk factor for IVF failure.

Our results might provide helpful insights into the relationships between mtDNA mitochondrial DNA haplogroups or variants and TFF. However, 
functional studies with larger sample sizes are needed to substantiate these findings further. Furthermore, there is a limitation in that these results are not suitable to be generalized due to the cohort consisting of Han Chinese patients only. The $\mathrm{PI} 3 \mathrm{~K} / \mathrm{AKT}$ pathway is an intracellular signalling pathway important in regulating diverse cellular functions [14, 22], and the relationship between PI3K/Akt and the fertilisation ability of sperm should be investigated in the future. In addition, a recent study showed that polyunsaturated fatty acids could induce apoptosis of colon cancer cells through a mitochondrial dependent pathway [23], which might provide a new insight into the mechanism of TFF.

In conclusion, ND5 and ND6 gene variations were correlated with TFF. Haplogroup R and haplogroup D4a were risk factors for TFF. This study indicated that the detection of sperm mtDNA variants and haplogroups might provide an accurate assessment of sperm fertilisation ability, and might be essential for the appropriate treatment of patients and for preventing the occurrence of TFF.

\section{Acknowledgments}

The authors report no financial or commercial conflicts of interest. This work was supported by the Health Department of Henan Province, China (Grant 4117), the Foundation of the He'nan Educational Committee (Grant 13A320637) and the science and technology project of Henan Province.

\section{Conflict of interest}

The authors declare no conflict of interest.

\section{References}

1. Zhu J, Jiang H, He RB, et al. Association between etiologic factors in infertile couples and fertilization failure in conventional in vitro fertilization cycles. Andrology 2015; 3: 717-22

2. Peultier AS, Fréour T, Cazenave N, Barrière P. Fertilization failure in IVF and ICSI. J Gynecol Obstet Biol Reprod 2015; 44: 380-6.

3. Mahutte NG, Arici A. Failed fertilization: is it predictable? Curr Opin Obstet Gynecol 2003; 15: 211-8.

4. Vanden Meerschaut F, Nikiforaki D, Heindryckx B, De Sutter P. Assisted oocyte activation following ICSI fertilization failure. Reprod Biomed Online 2014; 28: 560-71.

5. Barlow $P$, Englert $Y$, Puissant $F$, et al. Fertilization failure in IVF: why and what next? Hum Reprod 1990; 5: 451-6.

6. Hunte C, Zickermann V, Brandt U. Functional modules and structural basis of conformational coupling in mitochondrial complex I. Science 2010; 329: 448-51.

7. Fromm S, Senkler J, Eubel H, Peterhänsel C, Braun HP. Life without complex I: proteome analyses of an Arabidopsis mutant lacking the mitochondrial NADH dehydrogenase complex. J Exp Bot 2016; 67: 3079-93.

8. Mao GH, Wang YN, Xu M, Wang WL, Tan L, Tao SB. Polymorphisms in the MT-ATP6 and MT-CYB genes in in vitro fertilization failure. Mitochondrial DNA 2015; 26: 20-4.
9. Mao G, Lu P, Huang XH, et al. The analysis of mitochondrial DNA haplogroups and variants for in vitro fertilization failure in a Han Chinese population. Mitochondrial DNA A DNA Mapp Seq Anal 2016; 27: 2993-3000.

10. Gusdon AM, Votyakova TV, Reynolds IJ, Mathews CE. Nuclear and mitochondrial interaction involving mt-Nd2 leads to increased mitochondrial reactive oxygen species production. J Biol Chem 2007; 282: 5171-9.

11. Bungum M, Giwercman A, Bungum L, Humaidan P, Rastkhani H, Giwercman YL. Polymorphisms in the protein C inhibitor gene in in vitro fertilization failure. Fertil Steril 2010; 93: 277-9.

12. Kloss-Brandstätter A1, Schäfer G, Erhart G, et al. Somatic mutations throughout the entire mitochondrial genome are associated with elevated PSA levels in prostate cancer patients. Am J Hum Genet 2010; 87: 802-12.

13. Tian M, Bao H, Martin FL, et al. Association of DNA methylation and mitochondrial DNA copy number with human semen quality. Biol Reprod 2014; 91: 101.

14. Zhu X, Shi D, Li X, et al. TLR signalling affects sperm mitochondrial function and motility via phosphatidylinositol 3-kinase and glycogen synthase kinase-3alpha. Cell Signal 2016; 28: 148-56.

15. Mi Y, Shi Z, Li J. Spata19 is critical for sperm mitochondrial function and male fertility. Mol Reprod Dev 2015; 82: 907-13.

16. Condorelli RA, La Vignera S, Bellanca S, Vicari E, Calogero AE. Myoinositol: does it improve sperm mitochondrial function and sperm motility? Urology 2012; 79: 1290-5.

17. Sauna ZE, Kimchi-Sarfaty C. Understanding the contribution of synonymous mutations to human disease. Nat Rev Genet 2011; 12: 683-91.

18. Chen R, Davydov EV, Sirota M, Butte AJ. Non-synonymous and synonymous coding SNPs show similar likelihood and effect size of human disease association. PLoS One 2010; 5: e13574.

19. Weiss H, Friedrich T, Hofhaus G, Preis D. The respiratory-chain NADH dehydrogenase (complex I) of mitochondria. Eur J Biochem 1991; 197: 563-76.

20. Marchetti C, Obert G, Deffosez A, Formstecher P, Marchetti P. Study of mitochondrial membrane potential, reactive oxygen species, DNA fragmentation and cell viability by flow cytometry in human sperm. Hum Reprod 2002; 17: 1257-65.

21. Perl A, Qian Y, Chohan KR, et al. Transaldolase is essential for maintenance of the mitochondrial transmembrane potential and fertility of spermatozoa. Proc Natl Acad Sci USA 2006; 103: 14813-8.

22. Yang S, Li H, Tang L, et al. Apelin-13 protects the heart against ischemia-reperfusion injury through the RISKGSK-3beta-mPTP pathway. Arch Med Sci 2015; 11: 1065-73.

23. Zhang C, Yu H, Shen Y, Ni X, Shen S, Das UN. Polyunsaturated fatty acids trigger apoptosis of colon cancer cells through a mitochondrial pathway. Arch Med Sci 2015; 11: 1081-94. 